Treg. This may point towards a regulatory role of the noncanonical NF- $\kappa \mathrm{B}$ pathway in RA synovial inflammation, which could be exploited for the development of future new therapies.

\section{A149 NUCLEAR FACTOR- $\kappa$ B (NF- $\kappa$ B) INDUCING KINASE IS PREFERENTIALLY EXPRESSED IN RHEUMATOID ARTHRITIS SYNOVIAL TISSUE CONTAINING ECTOPIC LYMPHOID NEOGENESIS}

A-R Noort, K P M van Zoest, M Modesti, S W Tas, P P Tak Academic Medical Center/ University of Amsterdam, The Netherlands

10.1136/ard.2010.129643z

Background and Objectives In approximately 30\% of patients with rheumatoid arthritis (RA), synovial inflammation is characterised by ectopic lymphoid neogenesis (ELN) resulting in 'germinal centre-like' structures that contain dendritic cells (DC) and aggregates of $B$ and $T$ cells. This process does not define a specific disease subset and also occurs in other types of chronic inflammation. Nuclear factor- $\kappa B$ (NF- $\mathrm{KB}$ ) transcription factors are essential for the expression of proinflammatory cytokines and they induce regulatory circuits. These regulatory properties are thought to be governed at least in part by the non-canonical NF- $\kappa B$ pathway of which NF- $\mathrm{BB}$-inducing kinase (NIK) is a key mediator. Non-canonical NF- $\kappa B$ signalling can be triggered by stimuli like CD40L and lymphotoxin that are abundantly present in ELN. In addition, non-canonical NF- $\kappa B$ signalling is involved in indoleamine-2,3-dioxygenase (IDO) expression in DC that can suppress $\mathrm{T}$ cell responses and promote tolerance. Recently it was demonstrated that these cells may play an important role in the balance between regulatory $T$ cells (Treg) and Th17 cells. We investigated the presence of regulatory mechanisms in RA synovial tissue (ST) in relationship to ELN. We therefore studied the expression and distribution of NIK, IDO, Foxp3 (as a marker of Treg) and interleukin (IL)17 expressing cells in ST with and without ELN.

Methods Using immunohistochemistry, we analysed the expression of NIK, IDO, Foxp3 and IL17 in ST of 40 patients with RA. ST was obtained via mini-arthroscopy of inflamed knee or ankle joints of RA patients with active disease. Expression was scored on a semiquantitative 5-point scale $(0-4)$ by two independent observers. Foxp3 expression was quantified by scoring the number of positive cells per HPF.

Results ELN was present in 15 out of 40 RA ST. NIK expression was significantly higher in ST with ELN and more abundantly present within lymphocyte aggregates $(1.53+0.32$ vs $0.62+0.19 ; \mathrm{p}<0.05)$. We also observed more Foxp3 positive cells in ST containing ELN $(6.02+1.37$ vs $4.36+1.31)$. IDO and IL17 expressing cells were found in ST with and without ELN, but no significant difference between the two types of synovial inflammation was found.

Conclusions NIK is preferentially expressed in RA ST containing ELN in association with a trend towards more 